\title{
Morphology of Dust Lanes in Bars
}

\author{
Michael W. Regan and Stuart N. Vogel \\ Department of Astronomy, University of Maryland, College Park, MD \\ 20742
}

\begin{abstract}
Given that dust and molecular gas tend to concentrate at the locations of either shocks or orbit crowding, the study of the dust morphology of barred spirals can give insight into the kinematics of the galaxies. In order to do this, we present a sample of barred galaxies for which we have obtained near infrared and optical photometry and BIMA interferometer observations of $\mathrm{CO}$ emission. We obtain the dust extinction and relative scale height of the dust and stars using a radiative transfer model to interpret the photometry. We find that the scale height of the stars compared to the dust is generally larger in the central regions compared to further out in the bar dust lanes. The morphology of the dust extinction regions closely resembles the distribution of $\mathrm{CO}$ emission. An analysis of a larger sample of galaxies with IR data indicates that dust column densities appear larger in the nuclear regions than in the bar dust lanes. The sample also shows that the distribution of dust is not symmetrical along the bar with one side of the bar usually having a larger column density of dust than the other side.
\end{abstract}

\section{Introduction}

There is a discrepancy between what models predict for the dust and gas distribution in barred spirals and what is observed with optical extinction and with millimeter interferometers observing CO emission. The optical observations of strongly barred spirals are dominated by the two straight offset dust lanes along the leading edges of the bar. The majority of the previous $\mathrm{CO}$ observations show a "twin peaks" structure (Kenney et al. 1992) where the CO emission has two peaks at the inner end of the dust lanes. Recent high resolution models of the gas flow in barred spirals predict that the highest column densities are in a nuclear ring of dust and gas (Piner, Stone, \& Teuben 1995).

In this paper we will try to resolve some of the discrepancy by using new $\mathrm{CO}$ and near infrared observations of a sample of barred spiral galaxies.

\section{Observations}

The near infrared observations were obtained on three different observing runs. The first set was obtained on the $1.3 \mathrm{~m}$ at Kitt Peak using the Simultaneous Quad Infrared Imaging Device (SQIID) in January 1994. The second set was obtained on the $1.3 \mathrm{~m}$ at Kitt Peak using the Cryogenic Optical Bench in March 


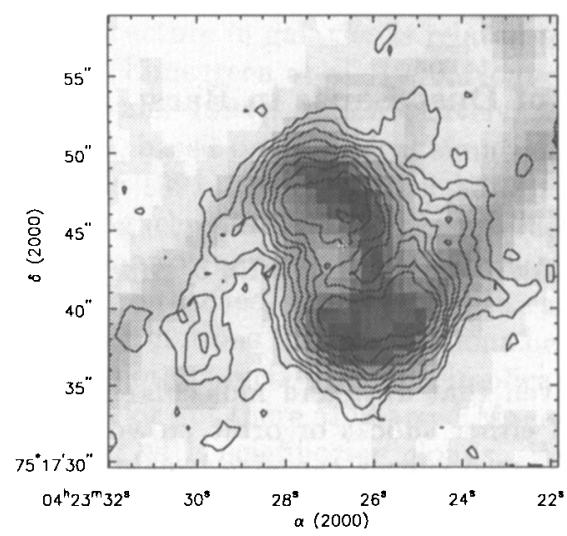

Figure 1. CO integrated brightness compared with $I-K$ color for NGC 1530. The contours are the BIMA CO $(\mathrm{J}=1-0)$ total intensity map and are at two sigma intervals. The beam is 5.2 by 4.5 with a position angle of $-74^{\circ}$. The gray scale image is the $I-K$ color variation. Here we can see that the $I-K$ color correlates with the $\mathrm{CO}$ total intensity although there are regions where there is extinction and no $\mathrm{CO}$.

1994. The third set were obtained on the $1.5 \mathrm{~m}$ at Cerro Tololo using the CTIO IR Imager (CIRIM).

The millimeter observations were obtained using the Berkeley- Illinois Maryland Array observing the $\mathrm{CO} \mathrm{J}=1-0$ transition. All the observations were made in three configurations of six antennas each yielding beams of approximately 4 arcseconds.

\section{Discussion}

One reason why the center of the galaxies would not show very much extinction in the optical would be if at optical wavelengths the dust optical depth is high enough to absorb most of the light from the stars on the far side of the galaxy. In this case the light near the bulge would be dominated by the unreddened stars on the near side of the galaxy leading to unreddened colors. By using colors that have $K$-band as their long wavelength band this effect can be avoided over a larger range of dust column depth.

By overlaying the CO map for NGC 1530 on the $I-K$ color map we can see that there is a strong correlation between the two (Figure 1). The strong correlation between near infrared extinction and $\mathrm{CO}$ emission is repeated for NGC 5383 (Figure 2). In addition, there is a strong asymmetry between the two sides of the bar. This asymmetry is seen in other galaxies within our sample. 


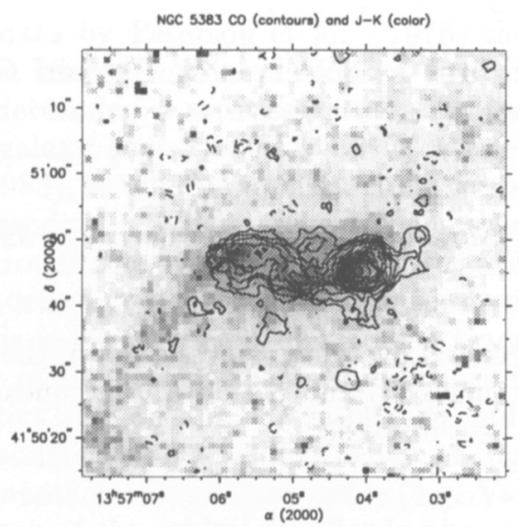

Figure 2. CO integrated brightness compared with $J-K$ color for NGC 5383. The contours are the BIMA CO $(\mathrm{J}=1-0)$ total intensity map and are at two sigma intervals. The beam is $4 . \prime 4$ by $4 . \prime 2$ with a position angle of $66^{\circ}$. The gray scale image is the $J-K$ color variation from SQIID and COB observations. In this galaxy the correlation between $\mathrm{CO}$ and $J-K$ color is very strong. Both show a triple peak structure with two of the peaks being at the end of the dust lanes and one of the peaks being on the nucleus. As in NGC 2903 and NGC 4314, one of the dust lane ends is much stronger than the other.

\section{Conclusions}

We have shown why optical colors are poor tracers of dust column depths since at reasonable dust column depths the dust becomes optically thick to light at optical wavelengths leaving the near-side stars dominating the light. By using $K$-band in a color it is possible to come up with a more reliable tracer of the dust column density.

By using the near infrared color maps we have shown how the dust extinction maps agree quite well with the $\mathrm{CO}$ velocity integrated brightness maps. We have also seen that both of these maps show that the gas and dust column densities are much higher in the rings and "twin peaks" near the nucleus than in the bar dust lanes.

It is also clear from the observations that the dust and gas have a much stronger $m=1$ component than the stellar potential traced by the stellar light distribution.

\section{References}

Kenney, J. D. P., Wilson, C. D., Scoville, N. Z., Devereux, N. A., \& Young, J. 1992, ApJ, 395, L79

Piner, B. G., Stone, J. M., \& Teuben, P. J. 1993, BAAS, 25, 1411 